\title{
Identification of hypoxic gene-signature as a prognostic and predictive biomarker to determine effective therapy in high risk bladder cancer patients
}

\author{
Zheng Tan, Ninadh M. D'Costa, Peter A. Raven, Claudia Chavez-Munoz, Alan I. So \\ Department of Urologic Sciences, University of British Columbia, Vancouver, BC, Canada \\ Correspondence to: Alan I. So. Department of Urologic Sciences, University of British Columbia, Level 6, 2775-Laurel St, Vancouver, BC V5Z1M9, \\ Canada. Email: alan.so@ubc.ca. \\ Provenance: This is a Guest Editorial commissioned by Section Editor Xiao Li (Department of Urologic Surgery, The Affiliated Cancer Hospital of \\ Jiangsu Province of Nanjing Medical University, Nanjing, China). \\ Comment on: Yang L, Taylor J, Eustace A, et al. A Gene Signature for Selecting Benefit from Hypoxia Modification of Radiotherapy for High-Risk \\ Bladder Cancer Patients. Clin Cancer Res 2017;23:4761-8.
}

Submitted Dec 04, 2017. Accepted for publication Dec 12, 2017.

doi: $10.21037 /$ tau.2017.12.17

View this article at: http://dx.doi.org/10.21037/tau.2017.12.17

Bladder cancer is the ninth most commonly diagnosed cancer in the world and fifth in the USA and Canada $(1,2)$. Patients presenting with bladder cancer are initially treated with transurethral resection and specimens are graded and staged. Muscle-invasive bladder cancers (MIBC) considered $>$ pT2, are currently treated with radical cystectomy with neoadjuvant chemotherapy or a combination of radiation and chemotherapy (3-6).

Tumor hypoxia, which is common in multiple cancer types $(7,8)$, is a biological condition that is characterized by deficient tissue oxygenation compromising biological functions. It interferes with the curability of solid tumors, regardless of the treatment modality employed (9). It is well-known that bladder cancer tumors, like other solid tumors, contain hypoxic areas and high expression of hypoxia-inducible markers have been associated with poor prognosis (10). There is good evidence that hypoxic tumors benefit from hypoxia-modifying therapy (11-13). However, there are no validated biomarkers that can select MIBC patients that would benefit from adding hypoxia-modifying therapy to radiotherapy. Although hypoxia gene signatures have been developed for head and neck, breast, and lung cancers, none have been developed for bladder cancer (12-14). In fact, the bladder carbogen and nicotinamide $(\mathrm{BCON})$ phase III clinical trial showed that the addition of hypoxia-modifying therapy (carbogen and nicotinamide) to radiotherapy improved overall survival. Therefore, Yang and collaborators proposed to identify a hypoxia gene signature for MIBC patients, which predicted benefit from hypoxia-modifying therapy. To achieve this, the research group analyzed all the published gene signatures available in the literature. Contradictory to what has been published, Yang and collaborators found that only the Lendahl hypoxic signature (15) was predictive of benefit from hypoxic modification but not prognostic in MIBC, confirming the need to identify a hypoxia gene signature exclusive for MIBC patients. To derive the bladder cancer-specific hypoxia signature, Yang analyzed 611 generic hypoxia genes. He hypothesized that a candidate gene would likely be hypoxia regulated in the bladder if coexpressing with multiple candidate genes. Therefore, the group developed a bladder cancer-specific hypoxia gene coexpression network. The network comprised 168 candidate hypoxia genes with 458 significant interactions. They determined that the higher number of interactions between the candidate genes indicated the likelihood of hypoxia relevance in bladder cancer. In the end they found 24 highly expressed hypoxia genes which were significantly associated with poor prognosis. Comparisons of the 24-hypoxia gene signature with other two published hypoxia gene signatures showed a 4 gene overlap (CAV1, P4HA2, DPYSL2, SLC2A3) with Chi et al. study (16), and 2 gene overlap (SLC16A1 and LDLR) with the head and neck signature which previously had shown to be prognostic in multiple cancer types(14). The 
authors discuss that the heterogeneity between signatures could be due to different biological pathways involved in tumor hypoxia in different cancer types, but also result from differences in the methods for obtaining these signatures.

Moreover, to investigate whether copy number variation biased the strength of gene-gene interactions, a null distribution was constructed to calculate the correlation values for 10,000 random gene-sets, of the same size as the gene pool. The correlations of the 24-gene signature were not significantly higher than the random sets $(\mathrm{P}=0.99)$, suggesting that the co-expression level was not driven by copy number variation. A similar analysis was performed to verify methylation data. It was found that the methylation rate of this 24-gene signature was significantly lower than that of random gene sets $(\mathrm{P}=0.0008)$, suggesting methylation status plays an important role in the computed co-expression level.

Furthermore, the de novo 24-gene signature was validated in several independent publically available cohorts comprising 679 fresh frozen tissue samples as well as in the BCON cohort of formalin-fixed paraffin embedded samples. Among BCON cohorts, protein expression data of three significant hypoxia biomarkers: carbonic anhydrase IX (CAIX), hypoxia-inducible factor 1-alpha (HIF-1 $\alpha)$ and glucose transporter 1 (GLUT1) were analyzed and a $t$-test (two tailed) was performed. The data showed that the 24gene signature score was significantly higher in tumors with high CAIX protein expression ( $\mathrm{P}=0.013$, upper quartile) and high HIF- $1 \alpha$ protein expression ( $\mathrm{P}=0.081$, lower quartile). However, no significant association was detected between the signature score and GLUT1 expression. In order to assess the prognostic and predictive performance in a more complex system, they studied the role of CAIX, HIF-1 $\alpha$, GLUT1 and necrosis within the BCON cohorts. Their analysis confirmed that tumors with high CAIX expression had poorer LPFS with RT alone ( $\mathrm{n}=64, \mathrm{P}=0.022$; HR, 2.21; 95\% CI, 1.12-4.37) but benefited from CON ( $\mathrm{n}=32$, $\mathrm{P}=0.017$; HR, 0.32; 95\% CI, 0.13-0.82). High GLUT1 expression had no prognostic value in patients treated with only RT $(\mathrm{P}=0.20)$ while there was a predictive significance in patients treated with $\mathrm{RT}+\mathrm{CON}(\mathrm{n}=48, \mathrm{P}=0.077$; HR, $0.51 ; 95 \%$ CI, 0.24-1.08). There was no prognostic or predictive significance associate with HIF-1 $\alpha$ expression. Necrosis was associated with poor prognosis $(\mathrm{n}=75$, $\mathrm{P}=0.029 ; \mathrm{HR}, 1.97 ; 95 \% \mathrm{CI}, 1.08-3.60)$ and good predictive value $(\mathrm{n}=80, \mathrm{P}=0.0051 ; \mathrm{HR}, 0.43 ; 95 \% \mathrm{CI}, 0.24-0.78)$ with a significant interaction $(\mathrm{P}=0.002)$. These assessments indicated that by combining necrosis and the gene signature score, a wider range of patients can be identified to benefit from hypoxia-modifying radiotherapy.

The current study not only successfully identified a de novo 24-gene signature in muscle invasive bladder cancer but also analyzed its correlation with other protein biomarkers to determine the prognostic and predictive value upon different tumor hypoxic levels. The authors also performed both multivariate and univariate analyses on the BCON cohort, evaluating any possible associations or interactions between each variable in bladder cancer progression (such as gender, age, tumor stage, etc.) and demonstrating no effects on the 24-gene signature. In conclusion, a hypoxia gene signature for muscle invasive bladder cancer patients was derived. The results from this study provide new clinical approaches to determine bladder cancer patients who would benefit from specific therapies including hypoxia modifying therapy. This study is an exemplary approach towards implementation of personalized medicine and integration of clinical studies into clinical practice.

\section{Acknowledgements}

None.

\section{Footnote}

Conflicts of Interest: The authors have no conflicts of interest to declare.

\section{References}

1. Ferlay J, Shin HR, Bray F, et al. Estimates of worldwide burden of cancer in 2008: GLOBOCAN 2008. Int J Cancer 2010;127:2893-917.

2. Statistics CCSsCoC. Canadian Cancer Statistics 2017. Toronto, ON: Canadian Cancer Society, 2017.

3. Gupta S, Gill D, Poole A, et al. Systemic Immunotherapy for Urothelial Cancer: Current Trends and Future Directions. Cancers (Basel) 2017;9.

4. von der Maase H, Sengelov L, Roberts JT, et al. Longterm survival results of a randomized trial comparing gemcitabine plus cisplatin, with methotrexate, vinblastine, doxorubicin, plus cisplatin in patients with bladder cancer. J Clin Oncol 2005;23:4602-8.

5. De Santis M, Bellmunt J, Mead G, et al. Randomized phase II/III trial assessing gemcitabine/carboplatin and methotrexate/carboplatin/vinblastine in patients with 
advanced urothelial cancer who are unfit for cisplatinbased chemotherapy: EORTC study 30986. J Clin Oncol 2012;30:191-9.

6. Bellmunt J, Théodore C, Demkov T, et al. Phase III trial of vinflunine plus best supportive care compared with best supportive care alone after a platinum-containing regimen in patients with advanced transitional cell carcinoma of the urothelial tract. J Clin Oncol 2009;27:4454-61.

7. Moeller BJ, Richardson RA, Dewhirst MW. Hypoxia and radiotherapy: opportunities for improved outcomes in cancer treatment. Cancer Metastasis Rev 2007;26:241-8.

8. Buffa FM, Harris AL, West CM, et al. Large metaanalysis of multiple cancers reveals a common, compact and highly prognostic hypoxia metagene. $\mathrm{Br} \mathrm{J}$ Cancer 2010;102:428-35.

9. Walsh JC, Lebedev A, Aten E, et al. The clinical importance of assessing tumor hypoxia: relationship of tumor hypoxia to prognosis and therapeutic opportunities. Antioxid Redox Signal 2014;21:1516-54.

10. Ord JJ, Agrawal S, Thamboo TP, et al. An investigation into the prognostic significance of necrosis and hypoxia in high grade and invasive bladder cancer. J Urol 2007;178:677-82.

Cite this article as: Tan Z, D'Costa NM, Raven PA, ChavezMunoz C, So AI. Identification of hypoxic gene-signature as a prognostic and predictive biomarker to determine effective therapy in high risk bladder cancer patients. Transl Androl Urol 2018;7(Suppl 1):S104-S106. doi: 10.21037/tau.2017.12.17
11. Eustace A, Mani N, Span PN, et al. A 26-gene hypoxia signature predicts benefit from hypoxia-modifying therapy in laryngeal cancer but not bladder cancer. Clin Cancer Res 2013;19:4879-88.

12. Eustace A, Irlam JJ, Taylor J, et al. Necrosis predicts benefit from hypoxia-modifying therapy in patients with high risk bladder cancer enrolled in a phase III randomised trial. Radiother Oncol 2013;108:40-7.

13. Toustrup K, Sørensen BS, Nordsmark M, et al. Development of a hypoxia gene expression classifier with predictive impact for hypoxic modification of radiotherapy in head and neck cancer. Cancer Res 2011;71:5923-31.

14. Winter SC, Buffa FM, Silva P, et al. Relation of a hypoxia metagene derived from head and neck cancer to prognosis of multiple cancers. Cancer Res 2007;67:3441-9.

15. Lendahl U, Lee KL, Yang H, et al. Generating specificity and diversity in the transcriptional response to hypoxia. Nat Rev Genet 2009;10:821-32.

16. Chi JT, Wang Z, Nuyten DS, et al. Gene expression programs in response to hypoxia: cell type specificity and prognostic significance in human cancers. PLoS Med 2006;3:e47. 